\title{
A GENERALIZATION OF AN INEQUALITY OF ZYGMUND
}

\section{R. PEREtz}

\author{
Department of Mathematics \\ University of Michigan \\ Ann Arbor, MI $\mathbf{4 8 1 0 9}$
}

(Received October 17, 1990)

ABSTRACT. The well known Bernstein inequality states that if $D$ is a disk centered at the origin with radius $R$ and if $p(z)$ is a polynomial of degree $n$, then $\max _{z \in D}\left|p^{\prime}(z)\right| \leq \frac{n}{R} \max _{z \in D}|p(z)|$ with equality iff $p(z)=A Z^{n}$. However it is true that we have the following better inequality:

$$
\max _{z \in D}\left|p^{\prime}(z)\right| \leq \frac{n}{R} \max _{z \in D}|\operatorname{Re} p(z)|
$$

with equality iff $p(z)=A Z^{n}$.

This is a consequence of a general equality that appears in Zygmund [7] (and which is due to Bernstein and Szegö): For any polynomial $p(z)$ of degree $n$ and for any $1 \leq p<\infty$ we have

$$
\left\{\int_{0}^{2 \pi}\left|p^{\prime}\left(e^{i x}\right)\right|^{p} d x\right\}^{1 / p} \leq A_{p} n\left\{\int_{0}^{2 \pi}\left|\operatorname{Re} p\left(e^{i x}\right)\right|^{p} d x\right\}^{1 / p}
$$

where $A_{p}^{p}=\pi^{1 / 2} \frac{\Gamma\left(\frac{1}{2} p+1\right)}{\Gamma\left(\frac{1}{2} p+\frac{1}{2}\right)}$ with equality iff $p(z)=A Z^{n}$.

In this note we generalize the last result to domains different from Euclidean disks by showing the following: If $g\left(e^{i x}\right)$ is differentiable and if $p(z)$ is a polynomial of degree $n$ then for any $1 \leq p<\infty$ we have

$$
\left\{\int_{0}^{2 \pi}\left|g\left(e^{i \theta}\right) p^{\prime}\left(g\left(e^{i \theta}\right)\right)\right|^{p} d \theta\right\}^{1 / p} \leq A_{p} n \max _{\beta}\left\{\int_{0}^{2 \pi}\left|\operatorname{Re}\left\{p\left(e^{i \beta} g\left(e^{i \theta}\right)\right)\right\}\right|^{p} d \theta\right\}^{1 / p}
$$

with equality iff $p(z)=A z^{n}$.

We then obtain some conclusions for Schlicht Functions.

Key Words and Phrases: Bernstein inequality, Bernstein-Szegö inequality, Krzyz problem, Dirichlet kernel, trigonometric interpolation

1980 AMS SUBJECT CLASSIFICATION CODES: 30A10, 30C10 


\section{INTRODUCTION.}

The classical result of Bernstein as it appears in [2] is Bernstein Inequality. If $D$ is a Euclidean disk and $P$ is a polynomial of degree $n$ over $\mathbf{C}$, then

$$
\left\|p^{\prime}\right\|_{D} \leq \frac{n}{\operatorname{tr}(D)}\|p\|_{D}
$$

where $\|f\|_{D}=\sup _{D}|f(z)|$ and $\operatorname{tr}(D)$ is the transfinite diameter of $D$ (which is the disk's radius in this case).

This result was generalized to various directions. The following theorem appears in [1]. Let $0 \leq k \leq 1$ and let $E$ be a closed $k$-quasidisk, then

THEOREM. For any polynomial $P$ of degree $n$ we have

$$
\left|\frac{p\left(z_{1}\right)-p\left(z_{2}\right)}{z_{1}-z_{2}}\right| \leq c_{1} \frac{n^{1+k}}{\operatorname{tr}(E)}\|p\|_{E}, z_{1}, z_{2} \in E
$$

and

$$
\left\|p^{\prime}\right\|_{E} \leq c_{2} \frac{n^{1+k}}{\operatorname{tr}(E)}\|p\|_{E}
$$

where $c_{1}=2^{-k} e\left(\frac{\pi}{4}+1\right)$ and $c_{2}=2^{-k} e$.

Another direction of generalization arises naturally in the following:

Let $\beta$ be the class of all analytic functions $f(z)=\sum_{k=0}^{\infty} a_{k} z^{k}$ in $|z|<1$ such that $0<|f(z)|<1$. A problem posed by Krzyz [4] is to determine $A_{n}=\max _{\beta}\left|a_{n}\right|, n \geq 1 \quad[3]$. The conjecture (which is still unsolved) is that $A_{n}=\frac{2}{e}$ and that it is attained only by rotations of

$$
g_{n}(z)=\exp \left(-\frac{z^{n}-1}{z^{n}+1}\right) \text {. }
$$

Let $f(z)$ be an extremal function for $A_{n}$.

CONJECTURE. $|\mathrm{f}(0)| \leq \frac{1}{\mathrm{e}}$ and equality holds only for rotations of $\mathrm{g}_{\mathrm{n}}$.

A theorem which indicates that this conjecture may be true is:

THEOREM [5]. If $n=2 p+1$ and if $a_{1}=a_{3}=\cdots=a_{2 p-1}=0$, then $\left|a_{0}\right| \leq \frac{1}{e}$. Equality sign occurs iff $\left|a_{n}\right|=\frac{2}{e}$

The proof of this uses the following generalization of (1): Let $D(0,1)=\{z \in \mathbb{C}|| z \mid<1\}$ and let $p$ be any polynomial of degree $n$ over $\mathbb{C}$, then

$$
\left\|p^{\prime}\right\|_{D(0,1)} \leq n\|\operatorname{Re} p\|_{D(0,1)}
$$

This follows from an inequality of Zygmund [7].

THEOREM. For any polynomial $p$ of degree $n$ and for any $1 \leq p<\infty$ we have 


$$
\left\{\int_{0}^{2 \pi}\left|\mathrm{p}^{\prime}\left(\mathrm{e}^{\mathrm{ix}}\right)\right|^{\mathrm{p}} \mathrm{dx}\right\}^{1 / \mathrm{p}} \leq \mathrm{A}_{\mathrm{p}} \mathrm{n}\left\{\int_{0}^{2 \pi}\left|\operatorname{Re} \mathrm{p}\left(\mathrm{e}^{\mathrm{ix}}\right)\right|^{\mathrm{p}} \mathrm{dx}\right\}^{1 / \mathrm{p}}
$$

where

$$
A_{p}^{p}=\pi^{1 / 2} \frac{\Gamma\left(\frac{1}{2} p+1\right)}{\Gamma\left(\frac{1}{2} p+\frac{1}{2}\right)}
$$

and equality occurs in (5) iff $p(z)=A z^{n}$.

In this note we indicate a way to generalize (5) to domains $E$ other than $D(0,1)$ by using the same ideas as in Zygmund's proof applied to $p \circ g$ where $g$ is a quite general mapping $D(0,1) \rightarrow E$.

\section{RESULTS.}

THEOREM 1. Let $g$ be a complex valued function of $e^{i x}, 0 \leq x \leq 2 \pi$. Suppose that $\left\{\arg g\left(e^{i x}\right) \mid 0 \leq x \leq 2 \pi\right\} \geq[0,2 \pi / n]$ and that $\frac{d g\left(e^{i x}\right)}{d x}$ exists, then for any non-negative, non- decreasing convex function $\chi$, for any $\alpha \in \mathbb{R}$ and for any polynomial $P$ of degree $n$ over $\mathbb{C}$ we have

$$
\int_{0}^{2 \pi} x\left(n^{-1}\left|\operatorname{Im}\left\{e^{i \alpha} g\left(e^{i \theta}\right) p^{\prime}\left(g\left(e^{i \theta}\right)\right)\right\}\right|\right) d \theta \leq \max _{\beta}\left\{\int_{0}^{2 \pi} \chi\left(\left|\operatorname{Re}\left\{p\left(e^{i \beta} g\left(e^{i \theta}\right)\right)\right\}\right|\right) d \theta\right\}
$$

equality occurs in (7) iff $p(z)=A z$.

We remark that the consequences of Theorem 1 hold true even if the condition

$$
\left\{\arg g\left(e^{i x}\right) \mid 0 \leq x \leq 2 \pi\right\} \geq[0,2 \pi / n]
$$

is dropped.

We will indicate at the end of Section 4 how to prove that.

With the notations of Theorem 1 we have

THEOREM 2. If $1 \leq p<\infty$, then

$$
\left\{\int_{0}^{2 \pi}\left|g\left(e^{i \theta}\right) p^{\prime}\left(g\left(e^{i \theta}\right)\right)\right|^{p} d \theta\right\}^{1 / p} \leq A_{p} n \max _{\beta}\left\{\int_{0}^{2 \pi}\left|\operatorname{Re}\left\{p\left(e^{i \beta} g\left(e^{i \theta}\right)\right)\right\}\right|^{p} d \theta\right\}^{1 / p}
$$

with equality iff $p(z)=A z^{n}$.

As a consequence we derive an analogous theorem to (1),

THEOREM 3. If $E$ is a simply connected domain such that $0 \in E$, and if $G: D(0,1) \rightarrow E$ is a Riemann mapping normalized by $G(0)=0$, then for every $1 \leq \mathrm{p}<\infty$ and every $0 \leq \mathrm{r}<1$ we have

$$
\left\{\int_{0}^{2 \pi}\left|P^{\prime}\left(G\left(r e^{i \theta}\right)\right)\right|^{p} d \theta\right\}^{1 / p} \leq \frac{4 A_{p} n}{r\left|G^{\prime}(0)\right|} \max _{\beta}\left\{\int_{0}^{2 \pi}\left|\operatorname{Re}\left\{P\left(e^{i \beta} G\left(r e^{i \theta}\right)\right)\right\}\right|^{p} d \theta\right\}^{1 / p}
$$

This last inequality is not sharp. 
Returning to the function $g$ of Theorem 1 we add

COROLLARY.

$$
\begin{gathered}
\max _{\alpha}\left\{\int_{0}^{2 \pi} \chi\left(\left|\operatorname{Im}\left\{e^{i \alpha} g\left(e^{i \theta}\right)\right\}\right|\right) d \theta\right\}=\max _{\beta}\left\{\int_{0}^{2 \pi} \chi\left(\left|\operatorname{Re}\left\{e^{i \beta} g\left(e^{i \theta}\right)\right\}\right|\right) d \theta\right\} \\
\left\{\int_{0}^{2 \pi}\left|g\left(e^{i \theta}\right)\right|^{p} d \theta\right\}^{1 / p} \leq A_{p} \max _{\beta}\left\{\int_{0}^{2 \pi}\left|\operatorname{Re}\left\{e^{i \beta} g\left(e^{i \theta}\right)\right\}\right|^{p} d \theta\right\}^{1 / p}
\end{gathered}
$$

The last corollary can be seen directly, but, it shows that we cannot drop "max" on the right hand of the above inequalities since it is easy to find a $g$ such that $\|\operatorname{Re} g\|_{p} \leq 1$ while $\underset{p \rightarrow \infty}{\lim }\|g\|_{p}=\infty$.

\section{PREPARATIONS.}

Let $p(z)=c_{0}+c_{1} z+\cdots+c_{n} z^{n}$ be a polynomial of degree $n$, where $\mathrm{c}_{0} \in \mathbb{R}$. We denote

$$
S(z)=\frac{1}{2}(p(z)+\overline{p(z)}), \tilde{S}(z)=\frac{1}{2 \dot{i}}(p(z)-\overline{p(z)})
$$

Let $g$ be a complex valued function of $e^{i x}, x \in \mathbb{R}$ such that $\left\{\arg g\left(e^{i x}\right) \mid 0 \leq x \leq 2 \pi\right) \geq\left[0, \frac{2 \pi}{n}\right]$ and such that $\frac{d g}{d x}\left(e^{i x}\right)$ exists. We denote

$$
\begin{gathered}
g\left(e^{i x}\right)=R(x) e^{i \phi(x)}, R(x)=\left|g\left(e^{i x}\right)\right|, \phi(x)=\arg g\left(e^{i x}\right) \\
S(x, t)=C_{0}+\sum_{v=1}^{n} R^{v}(x)\left(a_{v} \cos v t+b_{v} \sin v t\right) \\
\tilde{S}(x, t)=\sum_{v=1}^{n} R^{v}(x)\left(a_{v} \sin v t-b_{v} \cos v t\right) \\
\text { where } c_{0}, a_{1}, \cdots, a_{n}, b_{1}, \cdots, b_{n} \in \mathbb{R}
\end{gathered}
$$

where the coefficients $a, b$ are such that

$$
S(x, \phi(x))=S\left(g\left(e^{i x}\right)\right), \tilde{S}(x, \phi(x))=\tilde{S}\left(g\left(e^{i x}\right)\right) .
$$

As in Zygmund we denote the modified Dirichlet kernel and it's conjugate kernel by $D_{n}^{*}(u), \tilde{D}_{n}^{*}(u)$ respectively. Thus

$$
\begin{aligned}
& D_{n}^{*}(u)=\frac{1}{2} \sum_{\nu=1}^{n-1} \cos v u+\frac{1}{2} \cos n u=\frac{\sin n u}{2 \tan \frac{1}{2} u} \\
& \tilde{D}_{n}^{*}(u)=\sum_{\nu=1}^{n-1} \sin v u+\frac{1}{2} \sin n u=(1-\cos n u) \frac{1}{2} \cot \frac{1}{2} u
\end{aligned}
$$

We will also need the zeros of $\cos n t$ 


$$
u_{v}=(2 v-1) \pi / 2 n, v=1,2, \cdots, 2 n
$$

$\phi_{2 n}(t)$ will be a step function which has jumps $\frac{\pi}{n}$ at the points $u_{v}$. By (3.6), (3.21) on pages 10,11 [7] we have

THEOREM (Zygmund)

$$
\begin{aligned}
& S(x, u)=a_{n} R^{n}(x) \cos n u+\frac{1}{\pi} \int_{0}^{2 \pi} S(x, t) D_{n}^{*}(t-u) d \phi_{2 n}(t) \\
& \tilde{S}(x, u)=a_{n} R^{n}(x) \sin n u+\frac{1}{\pi} \int_{0}^{2 \pi} S(x, t) \tilde{D}_{n}^{*}(t-u) d \phi_{2 n}(t)
\end{aligned}
$$

Thus for any real number $\alpha$ we have

$$
\begin{aligned}
S\left(g\left(e^{i x}\right)\right) \cos \alpha-\tilde{S}\left(g\left(e^{i x}\right)\right) \sin \alpha=a_{n} R^{n}(x) \cos [n \phi(x)+\alpha]+ \\
+\frac{1}{\pi} \int_{0}^{2 \pi} S(x, t)\left\{\frac{\sin [n(\phi(x)-t)+\alpha]-\sin \alpha}{2 \tan \frac{1}{2}(\phi(x)-t)}\right\} d \phi_{2 n}(t)
\end{aligned}
$$

\section{A PROOF OF THEOREM 1 .}

As in Zygmund, let $x_{0}$ be a root of $\sin [n \phi(x)+\alpha]$ such that $\cos \left[n \phi\left(x_{0}\right)+\alpha\right]=1$. We differentiate (19) with respect to $x$ and substitute $x=x_{0}$. By (12) we have

$$
\begin{aligned}
& \frac{d S}{d x}\left(g\left(e^{i x}\right)\right)=-\operatorname{Im}\left\{e^{i x} g^{\prime}\left(e^{i x}\right) p^{\prime}\left(g\left(e^{i x}\right)\right)\right\} \\
& \frac{d \tilde{S}}{d x}\left(g\left(e^{i x}\right)\right)=\operatorname{Re}\left\{e^{i x} g^{\prime}\left(e^{i x}\right) p^{\prime}\left(g\left(e^{i x}\right)\right)\right\}
\end{aligned}
$$

This takes care of the left hand side of (19). On the right hand side we first differentiate $R(x)$ and use:

$$
\begin{gathered}
\frac{R^{\prime}(x)}{R(x)}=-\operatorname{Im}\left\{\frac{e^{i x} g^{\prime}\left(e^{i x}\right)}{g\left(e^{i x}\right)}\right\}, \\
\frac{\partial}{\partial t}\{\tilde{S}(x, t)\}=\sum_{v=1}^{n} v R^{v}(x)\left(a_{v} \cos v t+b_{v} \sin v t\right), \\
\left.\frac{\partial \tilde{S}}{\partial t}\right|_{t=\phi(x)}=\operatorname{Re}\left\{g\left(e^{i x}\right) p^{\prime}\left(g\left(e^{i x}\right)\right)\right\}, \\
\left.\frac{\partial \tilde{S}}{\partial t}\right|_{t=\phi(x)}=\operatorname{Im}\left\{g\left(e^{i x}\right) p^{\prime}\left(g\left(e^{i x}\right)\right\},\right. \\
-\operatorname{Im}\left\{\frac{e^{i x g^{\prime}\left(e^{i x}\right)}}{g\left(e^{i x}\right)}\right\}\left\{\operatorname{Re}\left\{g\left(e^{i x}\right) p^{\prime}\left\{g\left(e^{i x}\right)\right\} \cos \alpha-\operatorname{Im}\left\{g\left(e^{i x}\right) p^{\prime}\left(g\left(e^{i x}\right)\right)\right\} \sin \alpha\right\}\right.
\end{gathered}
$$


294

R. PERETZ

We now differentiate $\phi(x)$ on the right hand side of (19). Using (3.22) on page 12 [7] we get

$$
\operatorname{Re}\left\{\frac{e^{i x_{0}} g^{\prime}\left(e^{i x_{0}}\right)}{g\left(e^{i x_{0}}\right)}\right\} \frac{1}{n} \sum_{\nu=1}^{2 n} \frac{(-1)^{\nu+1}+\sin \alpha}{4 \sin ^{2} \frac{1}{2}\left(\phi\left(x_{0}\right)-u_{\nu}\right)} S\left(x_{0}, u_{v}\right)
$$

where we have used $\phi^{\prime}\left(x_{0}\right)=\operatorname{Re}\left\{\frac{e^{i x_{0}} g^{\prime}\left(e^{i x_{0}}\right)}{g\left(e^{i x_{0}}\right)}\right\}$.

Combining (20), (21), (22) with (19) gives

$$
\begin{aligned}
& -\operatorname{Im}\left\{e^{i\left(x_{0}+\alpha\right)} g^{\prime}\left(e^{i x_{0}}\right) p^{\prime}\left(g\left(e^{i x_{0}}\right)\right)\right\}= \\
& -\operatorname{Im}\left\{\frac{e^{i x_{0}} g^{\prime}\left(e^{i x_{0}}\right)}{g\left(e^{i x_{0}}\right)}\right\} \operatorname{Re}\left\{e^{i \alpha} g\left(e^{i x_{0}}\right) p^{\prime}\left(g\left(e^{i x_{0}}\right)\right)\right\}+ \\
& +\operatorname{Re}\left\{\frac{e^{i x_{0}} g^{\prime}\left(e^{i x_{0}}\right)}{g\left(e^{i x_{0}}\right)}\right\} \frac{1}{n} \sum_{v=1}^{2 n} \frac{(-1)^{v+1}+\sin \alpha}{4 \sin ^{2} \frac{1}{2}\left(\phi\left(x_{0}\right)-u_{v}\right)} s\left(x_{0}, u_{v}\right)
\end{aligned}
$$

We now use the identity $\operatorname{Im}(A \cdot B)=\operatorname{Re}(A) \operatorname{Im}(B)+\operatorname{Im}(A) \operatorname{Re}(B)$ with

$$
\begin{gathered}
A=\frac{e^{i x_{0}} g^{\prime}\left(e^{i x_{0}}\right)}{g\left(e^{i x_{0}}\right)}, B=e^{i \alpha} g\left(e^{i x_{0}}\right) p^{\prime}\left(g\left(e^{i x_{0}}\right)\right) \text { and get finally } \\
\quad \operatorname{Im}\left\{e^{i \alpha} g\left(e^{i x_{0}}\right) p^{\prime}\left(g\left(e^{i x_{0}}\right)\right)\right\}=-\frac{1}{n} \sum_{v=1}^{2 n} \frac{(-1)^{v+1}+\sin \alpha}{4 \sin ^{2} \frac{1}{2}\left(\phi\left(x_{0}\right)-u_{v}\right)} S\left(x_{0}, u_{v}\right)
\end{gathered}
$$

This is a generalization of (3.22) on page 12 of [7]. Let

$$
\beta_{v}=\left|\frac{(-1)^{v+1}+\sin \alpha}{4 \sin ^{2} \frac{1}{2}\left(\phi\left(x_{0}\right)-u_{v}\right)}\right|, v=1,2, \cdots, 2 \mathrm{n}
$$

then

$$
\beta_{1}+\beta_{2}+\cdots+\beta_{2 n}=n^{2}
$$

We use (23) with $R\left(\theta+x-x_{0}\right) e^{1\left(\phi(\theta)+\phi(x)-\phi\left(x_{0}\right)\right)}$ in place of $g\left(e^{i x}\right)$ (see (13)) and get

$$
\left|\operatorname{Im}\left\{e^{i \alpha} g\left(e^{i \theta}\right) p^{\prime}\left(g\left(e^{i \theta}\right)\right)\right\} \leq \frac{1}{n} \sum_{\nu=1}^{2 n} \beta_{v}\right| \operatorname{Re}\left\{P\left(e^{i\left(u_{v}-\phi\left(x_{0}\right)\right)} g\left(e^{i \theta}\right)\right)\right\} \mid
$$

Using the assumptions on $\chi,(25)$ and applying Jensen's inequailty we get 


$$
\chi\left(n^{-1}\left|\operatorname{Im}\left\{e^{i \alpha} g\left(e^{i \theta}\right) p^{\prime}\left(g\left(e^{i \theta}\right)\right)\right\}\right|\right) \leq \frac{1}{n^{2}} \sum_{v=1}^{2 n} \beta_{v} \chi\left(\left|\operatorname{Re}\left\{P\left(e^{i\left(u_{v}-\phi\left(x_{0}\right)\right)} g\left(e^{i \theta}\right)\right)\right\}\right|\right)
$$
Integration with respect to $\theta$ gives (7). The equality assertion follows from Zygmund. This completes the proof of Theorem 1. a

To prove that the consequence of Theorem 1 hold true even if we drop the condition

$$
\left\{\arg g\left(e^{1 x}\right) \mid 0 \leq x \leq 2 \pi\right\} \geq[0,2 \pi / n]
$$

we can use $(3,23)$ in [7] with the following

$$
\mathrm{S}(\theta)=c_{0}+\sum_{1}^{n}\left(a_{v} \cos v \theta+b_{v} \sin v \theta\right) R^{\nu} \text { where } x_{0}=-\frac{\alpha}{n} .
$$

Then for $R \geq 0,0 \leq \theta, \alpha \leq 2 \pi$ we get

$$
\left|\operatorname{Im}\left(e^{i \alpha} \operatorname{Re} e^{i \theta} p^{\prime}\left(\operatorname{Re}^{i \theta}\right)\right)\right| \leq \frac{1}{n} \sum_{1}^{2 n} \beta_{v}\left|\operatorname{Re} p\left(\operatorname{Re}^{1\left(\theta+u_{k}+\frac{\alpha}{n}\right)}\right)\right|,
$$

where the $\beta_{v}$ are independent of $R, \theta$. From that we proceed as in the proof of Theorem 1 .

\section{A PROOF OF THEORDY 2 .}

$$
\begin{aligned}
& \text { Let } x(t)=t^{p} \text { in }(7) \text {. We get } \\
& \int_{0}^{2 \pi}\left|\operatorname{Im}\left\{e^{1 \alpha} g\left(e^{i \theta}\right) p^{\prime}\left(g\left(e^{i \theta}\right)\right)\right\}\right|^{p} d \theta \leq n^{p} \max _{\beta}\left\{\int_{0}^{2 \pi}\left|\operatorname{Re}\left\{P\left(e^{1 \beta} g\left(e^{1 \theta}\right)\right)\right\}\right|^{p} d \theta\right\}
\end{aligned}
$$

Let $g\left(e^{i \theta}\right) p^{\prime}\left(g\left(e^{i \theta}\right)\right)=A(\theta)+1 B(\theta)$ then we have

$$
\int_{0}^{2 \pi}|B(\theta) \cos \alpha+A(\theta) \sin \alpha|^{p} d \theta \leq n_{\beta}^{p}{ }_{\max }\left\{\int_{0}^{2 \pi}\left|\operatorname{Re}\left\{P\left(e^{i \beta} g\left(e^{i \theta}\right)\right)\right\}\right|^{p} d \theta\right\}
$$

As in Zygmund we integrate this with respect to $\alpha$ over $0 \leq \alpha \leq 2 \pi$, change the order of integration on the left hand side and use

$$
\int_{0}^{2 \pi}|a \cos \alpha+b \sin \alpha|^{P} d \alpha=\left(a^{2}+b^{2}\right)^{P / 2} \int_{0}^{2 \pi}|\sin \alpha|^{P} d \alpha
$$

to get

$$
\begin{aligned}
&\left\{\int_{0}^{2 \pi}\left|g\left(e^{i \theta}\right) p^{\prime}\left(g\left(e^{i \theta}\right)\right)\right|^{p} d \theta\right\}^{1 / p} \\
& \leq\left\{\frac{2 \pi}{\int_{0}^{2 \pi}|\sin \alpha|^{p} d \alpha}\right\}^{1 / p} n \max _{\beta}\left\{\int_{0}^{2 \pi}\left|\operatorname{Re}\left\{p\left(e^{i \beta} g\left(e^{1 \theta}\right)\right)\right\}\right|^{p} d \theta\right\}^{1 / p}
\end{aligned}
$$

this proves (8) and completes the proof of Theorem 2 . 
6. PROOFS OF THEOREA 3 AND THE COROLLARY.

By the normalization $G(0)=0$ we can use Theorem 2 with $g\left(e^{i x}\right)=G\left(r e^{i x}\right)$. We apply Koebe's $\frac{1}{4}$-theorem [6] to get $\frac{r\left|G^{\prime}(0)\right|}{4} \leq\left|G\left(r e^{i \theta}\right)\right|$. This bounds the left hand side of (8) from below and proves (9).

(10) follows from (7) with $p(z)=z$ applied to $g$ and to $1 g$.

(11) follows from (8) with $p(z)=z$.

\section{REFERENCES}

[1] Anderson, J.M., Gehring, F.W., Hinkkanen, A.: Polynomial Approximation in Quasidisks, in "Differential Geometry and Complex Analysis", edited by Chavel, I. and Farkas, H.M., Springer-Verlag, 1985. pp. 75-86.

[2] Cheney, E.W.: Introduction to approximation theory, McGraw-Hill, New York, 1966, p. 92.

[3] Hummel, J.A., Scheinberg, S., Zalcman, L.: A coefficient problem for bounded nonvanishing functions, Journal D' Analyse Math. Vol. 34 (1977), pp. 169-190.

[4] Krzyz, J.: Coefficient problem for bounded nonvanishing functions, Ann. Polon. Math. 20 (1968), p. 314.

[5] Peretz, R.: Some properties of extremal functions for Krzyz problem, accepted by $J$. of Complex Variables Theory and Applications.

[6] Pommerenke, Chr.: Univalent functions, Vandenhoeck and Ruprecht, Gottingen, 1975, p. 22.

[7] Zygmund, A.: Trigonometric Series, Cambridge Press, 1959, Vol. II, Chapter X. 


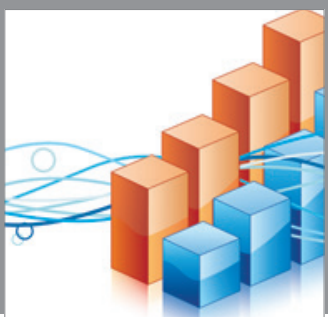

Advances in

Operations Research

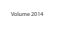

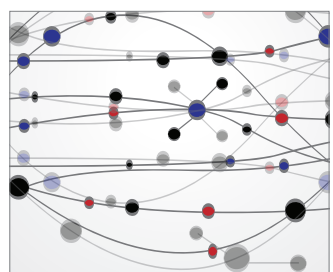

\section{The Scientific} World Journal
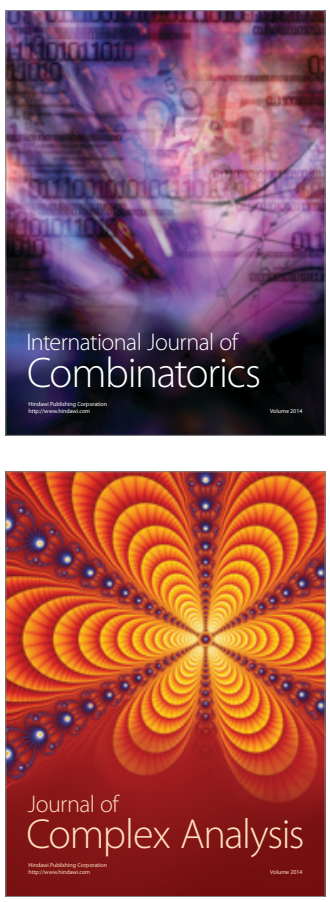

International Journal of

Mathematics and

Mathematical

Sciences
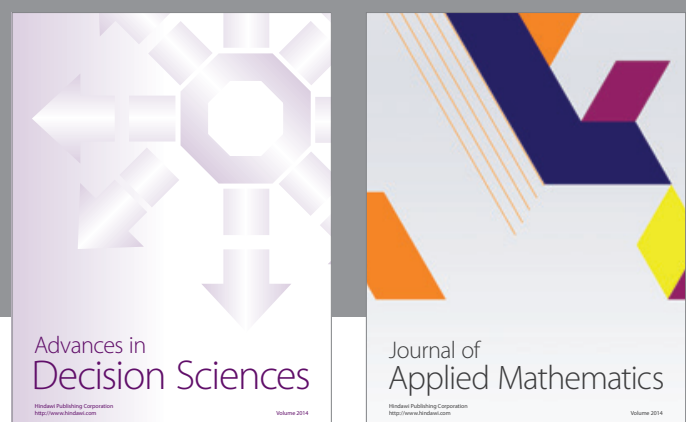

Journal of

Applied Mathematics
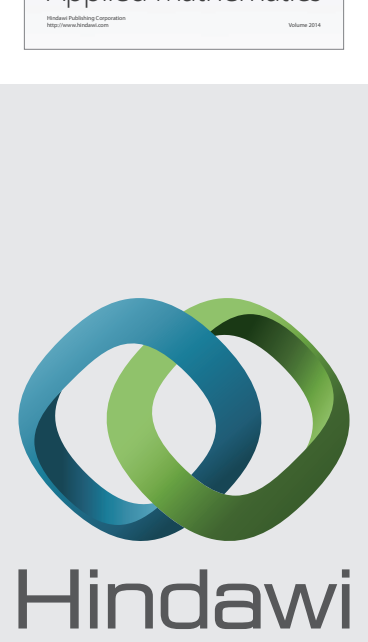

Submit your manuscripts at http://www.hindawi.com
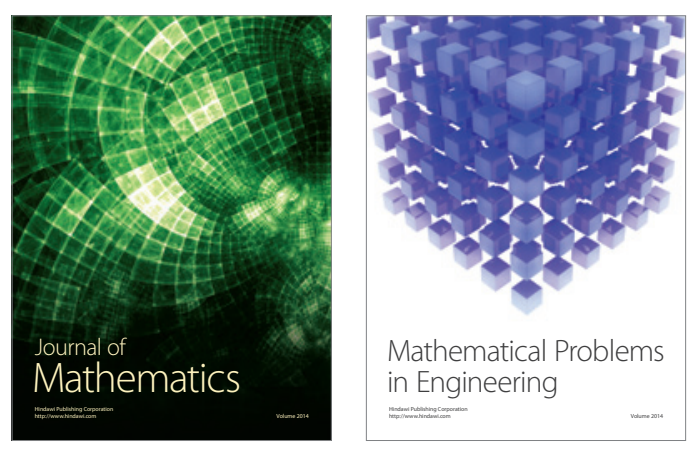

Mathematical Problems in Engineering
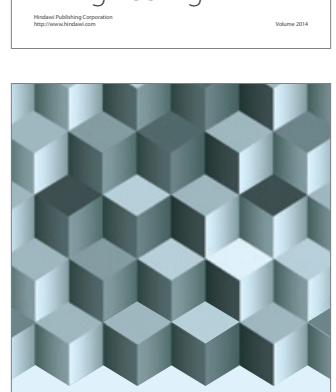

Journal of

Function Spaces
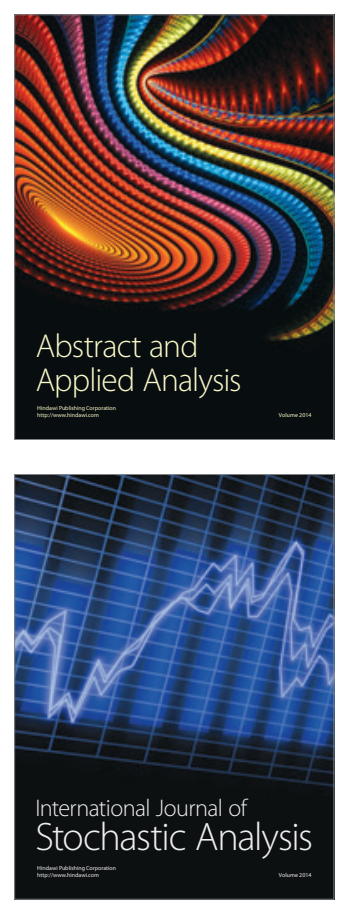

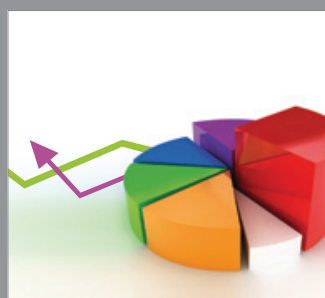

ournal of

Probability and Statistics

Promensencen
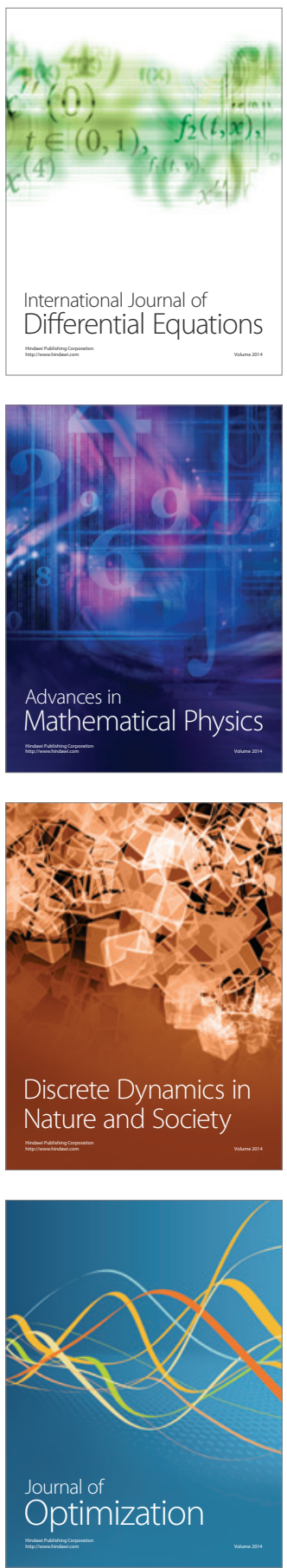